\title{
Distance based Cluster Head Selection Model for Optimum Number of Clusters in WSN
}

\author{
Namrata Arya \\ M. Tech. Scholar \\ Computer Science Engineering Dept. \\ VNS Cluster of Institutions, \\ Bhopal, India
}

\author{
Vivek Suryawanshi \\ Assistant Professor \\ Computer Science Engineering Dept. \\ VNS Cluster of Institutions, \\ Bhopal, India
}

\begin{abstract}
Wireless sensor network is growing very much in last few decades as its useful applications. To provide more functionality it's necessary to increase lifespan by clustering method. Clustering divides sensor nodes area into numbers of logical cluster. Each cluster has responsible node known as cluster head. This clustering and $\mathrm{CH}$ selection method uses lots of energy this energy can be saved by effective clustering method and improve lifespan of overall network. This work improves previous work for proper energy utilization for cluster head selection in WSN. Distance from base station is used for better clustering with optimum number of nodes within cluster. The nodes are categories into ring form with base station locate in center. A method with different probability of each node for cluster head selection is proposed. This probability of node select as cluster head is based on distance of current node from base station. The proposed method is reduces overall energy uses over other methods and performance is increased as numbers of nodes increases within large distance to base station
\end{abstract}

\section{Keywords}

Wireless Sensor network (WSN), Clustering, Energy consumption.

\section{INTRODUCTION}

A WSN is an arrangement of sensor hubs conveyed in a physical range and associated through remote connections. A sensor hub comprises of principally four units that are detecting, correspondence, preparing and control supply. The detecting hardware measures the distinctive parameters from the earth like temperature, dampness, weight and so on and changes over them into an electrical flag. Handling of such flags uncovers a few properties about the items or occasions happening in the encompassing of sensors. In the wake of preparing these signs can be transmitted to goal (base station) by utilizing radio transmitter either straightforwardly or through a halfway entryway. The fundamental components of a sensor system are self arranging ability, dynamic system topology, and restricted battery control, short range communicate correspondence, hubs portability, directing and extensive size of arrangement. Because of the capacity of self association and remote correspondence, sensor systems are relied upon to be utilized as a part of common, business and military applications, for example, reconnaissance, atmosphere and natural surroundings checking, vehicle following, debacle administration, restorative perception and acoustic information gathering. There are numerous difficulties in remote sensor systems. The key test is to amplify the soundness and also lifetime of system. It is not doable to supplant the batteries of hundreds or a great many sensor hubs after sending. In sensor organize, gathering of sensor hubs into a cluster is called bunching. Each cluster has a pioneer called bunch head. A bunch head might be pre alloted or chose by the individuals from the group. A cluster head gathers the information from the hubs inside bunch and exchange to goal (base station). The clustering methods generally examined by scientists increment the lifetime and adaptability goals. Numerous bunching conventions can be use to make various leveled structure that lessens the way cost when speaking with the base station.

In the level systems, total devours more vitality. Every hub shapes the tree by selecting the aggregator in course to sink, which causes an expansion in dormancy. Numerous scientists demonstrated that clustering strategy relieve the inertness. It enhances the system adaptability by making system working with disappointment of one $\mathrm{CH}$ [1]. The transmissions of a few information bundles to unified sink cause more vitality utilization i.e. progressively the information transmission the bigger is vitality utilization, and more is the data transmission prerequisite. The issue minimizes by diminishing the information parcels transmitted to sink by separating the whole system into gatherings and play out the Intra and entomb bunch total. In intra-cluster sensor hubs perform short range transmissions to the bunch head and have supreme directing structure. The added substance and separable information total capacity ( $\min , \max$, Avg, whole, check, middle and so on) at $\mathrm{CH}$ brings about the reduction of parcels to be imparted to sink which thus spares the vitality enhances the system lifetime and data transmission usage [2].

\section{CLASSIFICATION OF CLUSTERING PROTOCOLS}

In this segment we examine the arrangement of credits can be utilized to separate bunching conventions for remote sensor systems [3].

\subsection{Clustering Method}

The two essential methodologies for the co-appointment of whole clustering procedure are appropriated and unified. In disseminated clustering, where every sensor hub can run their own calculation and takes the choice of getting to be bunch head. In concentrated bunching, an incorporated power gathers the hubs to frame groups and cluster heads. Here and there mixture plan can likewise be executed.

\subsection{Cluster Properties}

In bunching approaches, there are a few qualities for the cluster arrangement. The accompanying are attributes that are identified with the inside structure of the bunch.

\subsubsection{Bunch tally}

Bunch tally is the quantity of groups shaped in a round. More number of cluster prompt to little size bunch dispersion, 
which is better in term of vitality utilization. In some bunching approaches, the choice of cluster heads are pre relegated from the conveyed sensor hubs for settled groups or bunch heads can be chosen haphazardly brings about factor number of groups.

\subsubsection{Cluster measure}

Cluster size is the greatest way length among the part hubs from bunch head. Little measured cluster is better in term of vitality utilization since it minimizes transmission separation and heap of bunch head. In some clustering approaches, bunch size is settled when bunch are altered for the duration of the life time, else it is variable for every bunch.

\subsubsection{Cluster Density}

Cluster thickness is characterized as extent of the quantity of bunch part in the bunch and cluster territory. There is enormous test to minimize the vitality utilization of bunch heads in thick groups. A portion of the bunching approach utilize settled clustering dependably has scanty thickness of bunch, yet in element clustering approaches cluster thickness variable.

\subsubsection{Message check}

Message tally is the quantity of message transmission is requiring for bunch head determination. More number of message transmission prompt to substantial measure of vitality utilization for bunch head determination strategy. There are numerous calculations which is non probabilistic, require the message transmission for bunch head choice.

\subsubsection{Security}

On the off chance that the individuals from a bunch are not altered the clustering plans are said to be versatile. Else we can consider as settled in light of the fact that the bunch check are not shifted all through the clustering procedure. The settled cluster tally builds the steadiness of a sensor organize.

\subsubsection{Intra-cluster topology}

It demonstrates the correspondence inside the cluster as immediate or multihop. It might be single jump or multihop from sensor hub to sensor hub or sensor hub to bunch head. However this correspondence likewise relies on upon the sensor's range. This constrained range limited the bunch head check.

\subsubsection{Between bunch head network}

It shows the abilities of sensor hubs/bunch makes a beeline for base station. On the off chance that the bunch heads are not having whole deal correspondence capacities, clustering plans needs to guarantee some middle of the road arrangement of steering to base station.

\subsection{Cluster Head Selection}

Bunch heads can be pre-relegated or picked arbitrarily from the conveyed set of hubs [3].

\subsubsection{Likelihood Based}

In likelihood based bunching calculations, every sensor hub utilizes pre relegated likelihood to decide the underlying cluster heads.

\subsubsection{Non Probability Based}

In no likelihood based bunching calculations more particular criteria for cluster head determination and bunch development are essentially considered which are chiefly in view of the sensor hubs nearness, network and degree and so forth.

\section{RELATED WORK}

Paper [11] proposed a novel Virtual Grid based Dynamic Routes Adjustment (VGDRA) plot that acquires minimum correspondence cost while keeping up about ideal courses to the most recent area of the versatile sink. This VGDRA plot parcels the sensor field into a virtual network and develops a virtual spine structure contained the cell-header hubs. A portable sink while moving around the sensor field continues changing its area and connects with the nearest marginal cellheader for information accumulation. Utilizing an arrangement of correspondence standards, just a predetermined number of the cell-headers partake in the courses recreation prepare in this way lessening the general correspondence cost. As far as hubs vitality utilization, the reproduction comes about uncover enhanced execution of our VGDRA conspire for various system sizes.

Paper [12] exhibited and assessed a novel plan for sorting out WSNs, in which numerous BSs are de-ployed however stand out BS is adaptively chosen to be dynamic. By utilizing the proposed plot, we productively use the transiently and spatially shifting vitality assets accessible to all BSs. Along these lines, the vast batteries and vitality reaping gadgets of individual BSs can be considerably decreased. To adaptively pick the dynamic BS, it gives a basic yet effective calculation HEF. We have demonstrated its asymptotic optimality under gentle conditions.

Another strategy proposed in paper [13] this method utilizes static clustering and $\mathrm{CHs}$ are chosen on the premise of the most extreme vitality of the hubs. This outcomes in settled number of $\mathrm{CHs}$ in each round and the ideal number of $\mathrm{CHs}$ is additionally kept up. We executed Packet Drop Model to make our convention more functional. We additionally actualized certainty interim to locate the conceivable deviation of our diagrams from the mean esteem, where mean esteem is ascertained by reenacting our convention 5 times and after that taking its mean. We contrast the consequences of our convention and that of the LEACH. REECH-ME beats LEACH in system lifetime, steadiness period, territory scope and throughput. Therefore, this plan upgrades the fancied qualities, i.e, least vitality utilization, most extreme solidness period, better lifetime and throughput distribute as contrasted and $\mathrm{LEACH}$.

\section{PROPOSED NETWORK MODEL 4.1 Assumptions}

To adjusts the proposed arrange demonstrate for gathering based information total the hub and system level suppositions are

\subsubsection{Hub Assumptions}

- Initially every one of the hubs have rise to vitality.

- Nodes in the system bunches as indicated by information in the parcel.

- $\mathrm{CH}$ chooses the connection of information parcels from the hubs, which are at one jump separate.

\subsubsection{Arrange Assumptions}

- The Sink, $\mathrm{CH}$ and hubs are static and homogeneous.

- All hubs in the cluster disperse consistently.

- Links between cluster head and hubs are unidirectional. 
- Clusters heads bunches as per amassed information parcels.

\subsection{Energy Model}

This study expect a straightforward model for the radio equipment where the transmitter disperses vitality for running the radio hardware to transmit and open up the signs, and the beneficiary runs the radio gadgets for gathering of signs [8]. Multipath blurring model (d4,power misfortune) for expansive separation transmissions and the free space show (d2,power misfortune) for proximal transmissions are considered. In this way to transmit a 1-bit message over a separation $\mathrm{d}$, the radio exhausts:

$$
\begin{gathered}
E_{T x}(l, d)=E_{T x-e l e c}(l)+E_{T x-a m p}(l, d) \\
E_{T x-e l e c}(l)=l E_{\text {elec }} \\
E_{T x-a m p}=l \epsilon_{f s} d^{2}, \text { when } d<d_{o} \\
E_{T x-a m p}=l \epsilon_{m p} d^{4}, \text { when } d \geq d_{o}
\end{gathered}
$$

To get a 1-bit message the collector consumes:

$$
E_{R x}(l)=l E_{\text {elec }}
$$

To total $\mathrm{n}$ data signs of length 1-bits, the vitality utilization was ascertained as:

$$
E_{D A-\text { expend }}=\ln E_{D A}
$$

The radio channel is thought to be symmetric, so the cost of transmitting a flag from A to B is same as that of transmitting a flag from B to A.

Table 1: Energy Model Parameters

\begin{tabular}{|c|c|}
\hline Parameter & Value \\
\hline Energy for data aggregation (EDA) & $5 \mathrm{~nJ} / \mathrm{bit} /$ signal \\
\hline Initial Node Energy & $0.5 \mathrm{~J}$ \\
\hline $\begin{array}{c}\text { Electronic Energy (Eelec) } \\
\text { Amplification energy for free space } \\
\text { model(Efs) }\end{array}$ & $10 \mathrm{pJ} / \mathrm{bit} / \mathrm{m} 2$ \\
\hline $\begin{array}{c}\text { Amplification energy for multi path } \\
\text { fading model (Emp) }\end{array}$ & $\begin{array}{c}0.0013 \\
\mathrm{pJ} / \mathrm{bit} / \mathrm{m} 4\end{array}$ \\
\hline $\begin{array}{c}\text { Threshold distance (d0) } \\
87 \mathrm{~m}\end{array}$ \\
\hline Packet Size (l) & 500 Bytes \\
\hline
\end{tabular}

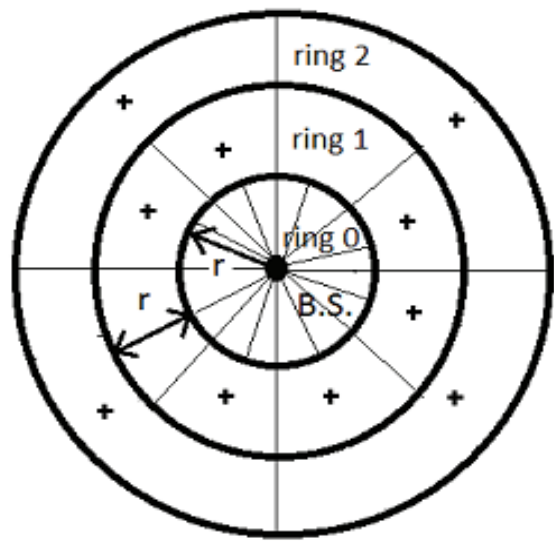

Fig 1: Proposed strategy roundabout system show
Fig. 1 demonstrates a roundabout system show that comprises of $\mathrm{M}(\mathrm{M}=3)$ concentric rings around a midway put Base Station. The bunches have been approximated to pie formed districts. "+" means a cluster head. Every round ring has a thickness of $r$, where $\mathrm{Mr}=$ Rnet. See that the quantity of bunches reductions as separation from the Base Station increments. Thusly, bunch measure diminishes as separation from the Base Station increments.

\section{NETWORK MODEL}

This area depicts the system display and other essential presumptions.

1. $\quad \mathrm{N}$ sensors are consistently scattered inside a round field $A_{\text {net }}$ of range span $R_{\text {net }}=\frac{A_{\text {net }}}{\pi}$. The Base Station is positioned at the focal point of the round area. The quantity of sensor hubs $\mathrm{N}$ to be sent depends particularly on the application.

2. The sensor hubs are thought to be stationary. Every sensor hub can speak with the Base Station straightforwardly.

3. Despite the fact that a Berkley sensor bit has more than 100 diverse power levels [9] persistent power levels for effortlessness as in [8] is accepted.

4. Correspondence is symmetric and a sensor can process the estimated remove in view of the got flag quality if the transmission power is known.

5. All sensors are area uninformed, i.e. not outfitted with GPS.

6. All sensors are homogeneous, i.e., they have similar limits.

All the sensor hubs have a specific identifier (ID) assigned to them. Every bunch head organizes the MAC and steering of parcels inside their groups. Consequently the bunches are synchronized by cluster heads impeccably and there is no loss of vitality while a sensor hub is conscious and sitting tight for its turn. The sensor system is partitioned into $M$ rings of equivalent thickness, $r$, Where $\mathrm{Mr}=\mathrm{R}$ (see Fig. 1). An information logging application is accepted where sensor hubs sense information and send it to their particular cluster heads. The bunch heads send information to the Base Station.

The rate of information being detected is thought to be uniform all through the sensor arrange. The medium is thought to be sans dispute and control messages between the cluster heads and the sensor hubs are not viewed as, expecting them to be short and present just a little overhead. Bunch heads total information in an impeccable way. Accordingly, bunch heads send just a solitary information bundle to the Base Station per round.

Table 1: Energy Model Parameters

\begin{tabular}{|l|l|}
\hline \multicolumn{1}{|c|}{ Parameter } & \multicolumn{1}{c|}{ Value } \\
\hline \multicolumn{1}{|c|}{ Symbol } & \multicolumn{1}{c|}{ Parameter Represented } \\
\hline$A_{n e t}$ & Area of sensor network \\
\hline$R_{n e t}$ & Radius of sensor network \\
\hline$N$ & Number of sensor nodes \\
\hline$M$ & Number of rings \\
\hline$r$ & Thickness of a ring \\
\hline
\end{tabular}




\section{DISTANCE BASED CH SELECTION MODEL}

A model called Distance Based $\mathrm{CH}$ Selection Model (DBCSM) where in the likelihood of a bunch head decision depends up on the separation of the sensor hub from the Base Station is proposed. Since sensor hubs are not outfitted with a GPS the Base Station sends a "welcome" message for start. Every sensor hub then approximates its separation (and in this way to which ring it has a place with) from the Base Station construct up in light of the got flag quality. The likelihood of race of a bunch head is given by the accompanying Eq.,

$$
P(C H)_{i}=\frac{k_{i}}{N_{i}}
$$

which is equal to,

$$
P(C H)_{i}=\frac{M^{2}}{R_{\text {net }}} \sqrt{\frac{3 \epsilon_{f s}(2 i+1)}{2 \epsilon_{m p}\left((i+1)^{6}-(i)^{6}\right) N}}
$$

Clusters are framed as like those in [7], where every sensor hub joins the nearest bunch head.

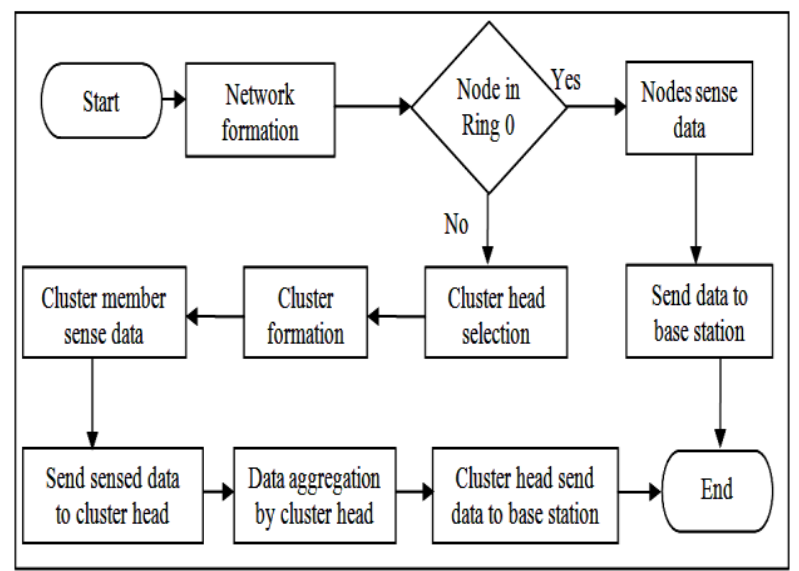

Figure 2. Flowchart for Proposed method

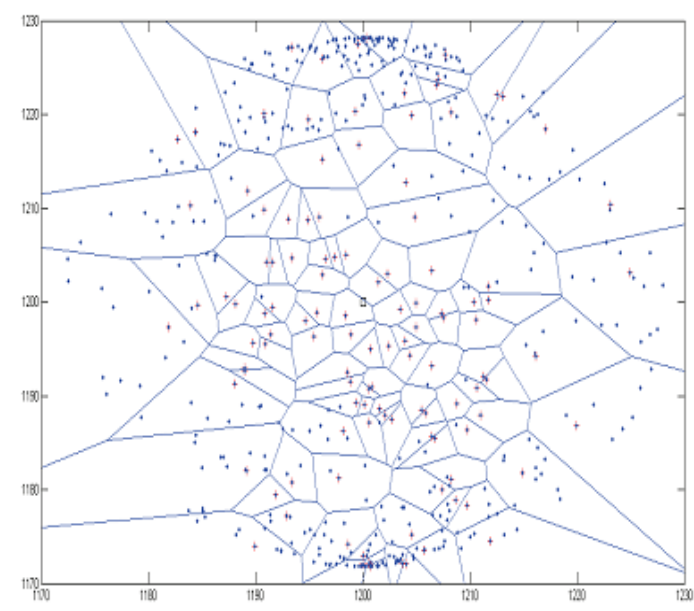

Figure 3: graphs for proposed method WSN

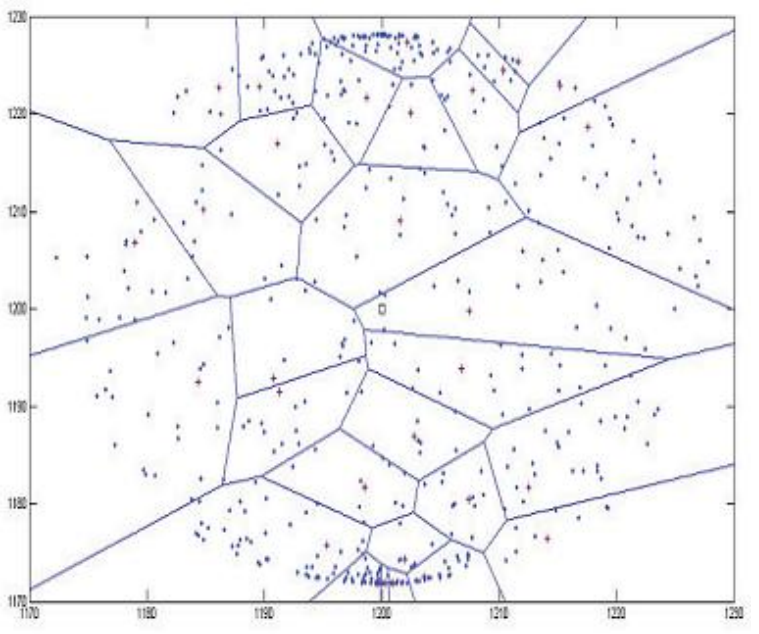

Figure 4: graphs for equal size cluster method WSN

\section{RESULTS EVALUATION}

In this area assessment of the DBCSM demonstrate has been finished. For correlation a model that chooses bunch heads with a uniform likelihood $(\mathrm{p}=0.05)$ is expected. This model is called Equal Probability Model (base technique) as in [10]. Both the models are contrasted with demonstrate that DBCSM prevails with regards to diminishing the vitality utilization in each ring around the Base Station. The vitality devoured by a bunch head and a sensor hub in the ith (i>1) around the Base Station is measured. The normal bunch estimate, both regarding span and number of hubs inside the group, is likewise systematically decided. The quantity of hubs $(\mathrm{N})$ considered are 500. The territory of the system (ANet) is $25 * 104 \mathrm{~m} 2$. The quantity of rings is 10 .

\subsection{Energy Usage}

The aggregate vitality utilization by sensor hubs per round for every ring is investigated as DBCSM minimizes the vitality use on a normal by around $21 \%$ (see Fig. 5) over equivalent cluster estimate strategy. Curiously the change in the vitality use in the rings nearest to the Base Station and uttermost far from the Base Station is the biggest. For the last two rings the vitality utilization is diminished by $34 \%$ and $46 \%$ separately. This demonstrates a bunching approach that chooses cluster heads consistently performs ineffectively in the rings that are either close or exceptionally far from the Base Station. The purpose behind higher vitality utilization is that equivalent cluster measure strategy chooses bunch heads with a uniform likelihood, which is suited for the normal case (the center rings).

For center (rings 3-6) the normal vitality spent per sensor hub in DBCSM is 0.96 that of equivalent cluster measure strategy. The rings that are nearer to the Base Station acquire misfortune when information is accumulated and got by the bunch heads. Sensor hubs nearer to the Base Station expend lesser vitality when transmitting information specifically to the Base Station. Misfortunes because of direct transmission are higher for rings that are more remote away. Another purpose behind change in the aggregate vitality devoured by sensor hubs in inward rings in DBCSM is the lower vitality utilization by cluster heads (see Fig. 6). The vitality utilization in the sensor hubs that are in external rings in DBCSM enhances because of the lesser number of cluster heads (see Fig. 13). 


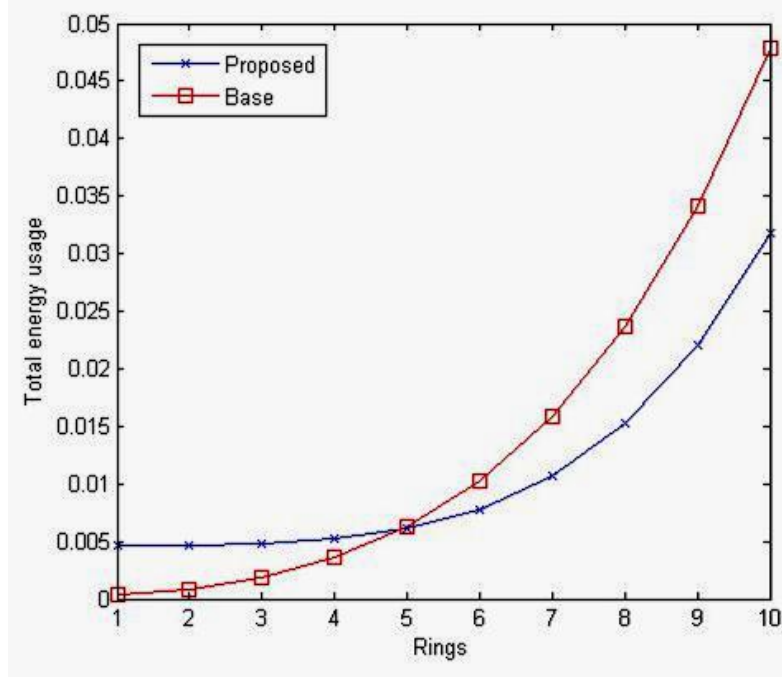

Fig 5: Demonstrates the aggregate vitality utilization for equivalent cluster measure technique and DBCSM for all the sensor hubs in every ring.

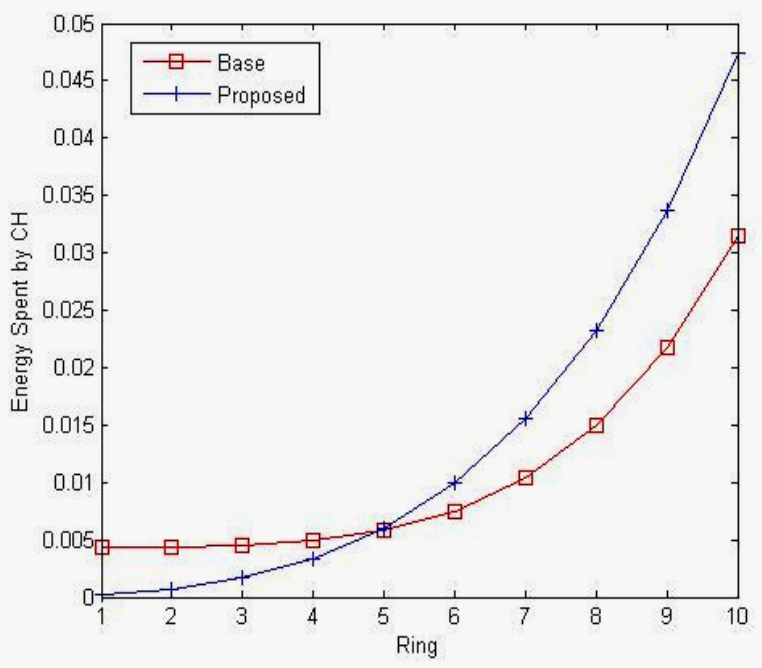

Fig 6: Demonstrates the vitality devoured by a solitary bunch set out toward both DBCSM and equivalent cluster measure strategy and around the Base Station for every ring.

\subsection{Energy Consumption by Cluster Heads}

The bunch heads in the rings nearer to the vase station devour lesser vitality than for DBCSM than those for equivalent cluster estimate technique allude fig. 6 . For the inward five (rings 1-5) the vitality devoured by a cluster head in DBCSM is 0.45 times that of equivalent bunch measure strategy. The reason essentially is lower cluster sizes for the rings that are nearer to the Base Station. This outcomes in lesser vitality utilization for accumulation and gathering of vitality. For ring 5 (practically the middle) the vitality utilization turns out to be about equivalent, strengthening our clarification that the ideal bunch head decision by equivalent cluster estimate strategy is for the rings with normal separation to the Base Station. For the rings that are more distant far from the Base Station, the normal vitality utilization is higher for DBCSM. For the external (rings 6-10) the normal vitality devoured by a cluster head in DBCSM is $48 \%$ higher than that for equivalent bunch estimate strategy. Be that as it may, the general vitality uses by all the sensor hubs in ring those away remains lower. This is on account of the quantity of bunch heads that are chosen are lower (see Fig. 7) for DBCSM. Consequently, lesser measure of information is transmitted to the Base Station and more information is privately gotten and accumulated into single transmission parcel. Such a plan would be extremely appropriate in applications where either the required exactness in information is low or the information has high repetition.

\subsection{Numbers of Cluster}

Fig 7 think about the measure of cluster framed by base technique and proposed strategy as number of bunches in every ring. The quantities of bunches in close to base station is almost twofold for proposed strategy. In any case, if hubs are close to base station then direct information transmission cost is lesser, so more cluster arrangement not prompts to high vitality misfortune. consequently separate from base station is builds number of cluster is abatements for proposed technique. The quantity of groups for external rings 6-10 is almost $65 \%$ lower than base technique. For base strategy number of groups increments as separation of base station increments.

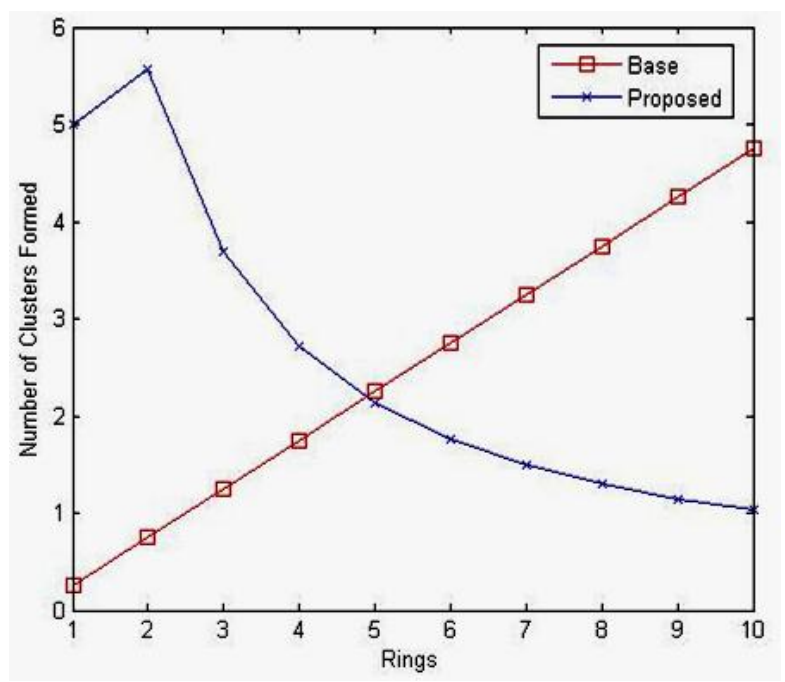

Fig 7: Shows the quantity of groups framed as an element of rings around the Base Station.

\section{CONCLUSION}

Clustering is a strategy to decrease vitality utilization and to give soundness in remote sensor systems. For heterogeneous remote sensor organizes, a few bunching conventions are proposed. A large portion of the late vitality effective bunching conventions intended for sensor systems depend on lingering vitality, normal vitality, area, thickness and so forth which are powerful in vitality sparing.

This work examines the issue of wasteful cluster head race in a remote sensor organize. The investigation demonstrates that decision of bunch heads with a uniform likelihood as in [8] prompts to wasteful vitality utilization. For this the sensor arrange has been separated into concentric rings $(M=10)$ around the Base Station. It is additionally demonstrated that for rings proximal to the Base Station more bunch heads $n$ be chosen (very nearly $200 \%$ ). For rings that are more remote far from the Base Station the lesser cluster heads ought to be chosen (just about 65\%).

A diagnostic model to foresee the quantity of cluster heads as a component of separation from the Base Station has been proposed. The model is called Distance Based CH Selection 
Model (DBCSM). In this work a hypothetical examination with equivalent bunch measure technique is finished. DBCSM on a normal gives $28 \%$ lessening in complete vitality use over equivalent cluster estimate technique. The examination recommends that higher number of cluster heads ought to be chosen nearer to the Base Station. As the separation from the Base Station builds the quantity of bunches framed reductions and thusly the cluster estimate increments.

\section{REFERENCES}

[1] Ameer Ahmed Abbasi, Mohamed Younis, "A survey on clustering algorithms for wireless sensor networks," Computer Communications, Volume 30, Issues 14-15, Network Coverage and Routing Schemes for Wireless Sensor Networks, 15 October 2007.

[2] Rajagopalan R, Varshney P.K, "Data-aggregation techniques in sensor networks: a survey, Communications Surveys \& Tutorials, IEEE , vol.8, no.4, pp.48-63, Fourth Quarter 2006.

[3] I.F. Akyildiz et al. 2002. Wireless sensor networks: a survey, Computer Network.

[4] Abdul Waheed Khan, Abdul Hanan Abdullah,, Mohammad Abdur Razzaque, , and Javed Iqbal Bangash, "VGDRA: A Virtual Grid-Based Dynamic Routes Adjustment Scheme for Mobile Sink-Based Wireless Sensor Networks", IEEE Sensors Journal, Vol. 15, No. 1, Pages 526-534, IEEE 2015.
[5] Runwei Zhang, Francois Ingelrest, Guillermo Barrenetxea, Patrick Thiran,Fellow, and Martin Vetterli, "The Beauty of the Commons: Optimal Load Sharing by Base Station Hopping in Wireless Sensor Networks", IEEE Journal On Selected Areas In Communications, Vol. 33, No. 8, Pages 1480-1491, IEEE, 2015

[6] A. Haider, N. Javaid, N. Amjad, A. A. Awan, A. Khan, N. Khan, "REECH-ME: Regional Energy Efficient Cluster Heads based on Maximum Energy Routing Protocol for WSNs", IEEE, 2013

[7] Ettus, M. (1998). System capacity, latency, and power consumption in multihop-routed SS-CDMA wireless networks. Radio and Wireless Conference, 1998. RAWCON 98. 1998 IEEE(pp. 55-58). IEEE press.

[8] Heinzelman, W., MA Chandrakasan, A., \& Balakrishnan, H. (2002). An application-specific protocol architecture for wireless microsensor networks. IEEE Transactions on Wireless Communications , 1(4), 660-670.

[9] Hill, J., Szewczyk, R., Woo, A., Hollar, S., Culler, D., \& Pister, K. (2000). System architecture directions for networked sensors. ACM Sigplan Notices , 35(11), 93104.

[10] Mhatre, V., \& Rosenberg, C. (2004). Homogeneous vs heterogeneous clustered sensor networks: a comparative study. IEEE International Conference on Communications, 2004. 6, pp. 3646-651. IEEE press. 